\title{
Stability of the zero solution of nonlinear differential equations under the influence of white noise
}

\author{
Irada Dzhalladova', Miroslava Růžičková2* and Viera Štoudková Růžičkováa
}

\section{"Correspondence:}

miroslava.ruzickova@fpv.uniza.sk

2University of Žilina, Žilina, Slovakia Full list of author information is

available at the end of the article

\section{Springer}

\begin{abstract}
The paper deals with a system of nonlinear differential equations under the influence of white noise. This system can be used as a mathematical model of various real problems in finance, mathematical biology, climatology, signal theory and others. Necessary and sufficient conditions for the asymptotic mean square stability of the zero solution of this system are derived in the paper. The paper introduces a new approach to studying such problems - construction of a suitable deterministic system with the use of Lyapunov function.
\end{abstract}

MSC: $34 \mathrm{~K} 50 ; 60 \mathrm{H} 10 ; 60 \mathrm{H} 30 ; 65 \mathrm{C} 30$

Keywords: stochastic systems; white noise; mean square stability; Lyapunov function

\section{Introduction}

We can come across stochastic behavior while examining many important problems of a global character in various fields of research, for example, in the theory of climate change. Detailed understanding of extreme events in climate, of phenomena that are beyond our normal expectations, is a very important topic in climatology, meteorology and related fields. Common methods of studying extreme events, such as the statistical approach, the empirical-physical approach or the numerical modeling approach, have some limitations, and study of them has been largely empirical.

The idea of replacing the whole deterministic system with a stochastic differential equation was introduced by Hasselmann in his work [1] on stochastic climate models that appeared in 1976. There he proposed to improve deterministic models for the 'climate' (slow variables) by incorporating the influence of the 'weather' (fast variables) in the form of random noise. The univariate linear systems that appear in the work have been successful in describing various modes of climate variability. Success of these models has inspired researchers to consider the stochastic forcing as a possible source of more complex dynamics, for example, in [2]. The direction of stochastic parametrizations in which the development of the climate models will be possible in the coming years is formulated, for example, in [3].

Hasselmann's works can be seen as the beginning of describing extreme events in climate by a stochastic system of differential equations in which random weather changes

(C) 2015 Dzhalladova et al.; licensee Springer. This article is distributed under the terms of the Creative Commons Attribution 4.0 International License (http://creativecommons.org/licenses/by/4.0/), which permits unrestricted use, distribution, and reproduction in any medium, provided you give appropriate credit to the original author(s) and the source, provide a link to the Creative Commons license, and indicate if changes were made. 
are expressed by a nonlinear stochastic perturbation in the form of white noise. Stochastic characteristics of white noise in the system are either known or are determined by the climate's median value.

Many works, dedicated to the study of evolution models, use white noise to express the amount of ice surface of the Arctic Ocean and then estimate the likelihood that it would spread on the entire surface of the Earth. Part of the mystery of the Earth's periodic ice ages was uncovered in connection with the so-called stochastic resonance that was first discovered while studying the periodic recurrence of Earth's ice ages, see $[4,5]$. Since its discovery, the stochastic resonance has been used in various experimental and theoretical studies. For example, it occurs in bi-stable systems, where the input-output relationship is nonlinear, the periodic input signal is weak and there is random, uncorrelated variation added to the signal of interest. In climatic models, the stochastic resonance manifests at its best when regular 'weak' weather changes are in certain proportion to the random weather fluctuations. By the term 'weak' we mean changes, that are not capable of causing climate changes on their own, but the presence of random weather changes causes disturbances in periodicity of the system, which then transforms into a different mode.

Our work deals with a stochastic model with nonlinear member. Some related problems regarding such systems were studied in [6]. In this paper we focus on the determination of conditions that are necessary and sufficient for stable behavior of the above mentioned processes. The present paper contains sufficient conditions for the asymptotic mean square stability of the zero solution of systems with white noise. Further, sufficient conditions for instability of the zero solution of this system and sufficient conditions for a more general system are derived in the paper. The results are followed by several examples. We construct Lyapunov functions as a tool to study stability of a stochastic system that works under the influence of white noise.

Let $(\Omega, \mathcal{F}, F, \mathbb{P})$ be a filtered probability space, or stochastic basis, consisting of a probability space $(\Omega, \mathcal{F}, \mathbb{P})$ and a filtration $F=\left\{\mathcal{F}_{t}, \forall t \geq 0\right\}$ contained in $\mathcal{F}$. On the probability space we consider the stochastic system of nonlinear differential equations of the form

$$
d x_{i}(t)=f_{i}(x) d t+h_{i}(x) d w_{i}(t), \quad i=1,2, \ldots, m, \quad t \geq 0,
$$

where the state function $x(t)=\left(x_{1}, \ldots, x_{m}\right)^{T}$ (the operation $T$ denotes transposition) is a continuously differentiable $m$-dimensional column vector-function, $f=\left(f_{1}, \ldots, f_{m}\right)^{T}$, $h=\left(h_{1}, \ldots, h_{m}\right)^{T}$ are also continuously differentiable $m$-dimensional column vectorfunctions such that $f(o)=o, h(o)=o, o=(0,0, \ldots, 0)^{T} \in \mathbb{R}^{m}$ hold. The function $f$ represents a slow deterministic process, the products $h_{i}(x) d w_{i}(t), i=1,2, \ldots, m$, are the stochastic approximations to a fast phenomenon. The $m$-dimensional column vector-function $w=\left(w_{1}, \ldots, w_{m}\right)^{T}$ indicates a standard Wiener process. The $m$-dimensional Wiener process is said to be standard Wiener process if

$$
\begin{aligned}
& w(0)=o, \\
& E^{(1)}\{d w(t)\}=o, \\
& E^{(1)}\left\{d w(t) d w^{T}(t)\right\}=I d t
\end{aligned}
$$


hold for $t \geq 0, I$ is the identity matrix. Any realization of the Wiener process $w(t)$ is continuous but not differentiable at each point. Moreover, because $E^{(1)}\left\{w(t) w^{T}(t)\right\}=I t$, the process $w(t)$ is a non-stationary stochastic process.

For simplicity, we denote $h(x) d w(t)=\left(h_{1} d w_{1}, \ldots, h_{m} d w_{m}\right)^{T}$ in our consideration. So, the product $h(x) d w(t)$ means neither a scalar product nor a vector product, but it is a column vector with components $h_{i}(x) d w_{i}(t), i=1,2, \ldots, m$. Using this, system (1) can be rewritten into the vector form

$$
d x(t)=f(x) d t+h(x) d w(t), \quad t \geq 0 .
$$

We are interested in stability of solutions of the system. There are several various definitions of stability that can be used. Here we recall the mean square stability and the asymptotic mean square stability of the zero solution.

Definition 1 The trivial solution of system (3) is said to be mean square stable on the interval $[0, \infty)$ if, for each $\varepsilon>0$, there exists $\delta>0$ such that any solution $x(t)$ corresponding to the initial data $x(0)$ exists for all $t \geq 0$ and the mathematical expectation

$$
E^{(1)}\left\{\|x(t)\|^{2}\right\}<\varepsilon \quad \text { whenever } t \geq 0 \text { and }\|x(0)\|<\delta .
$$

The mean stability of the zero solution of system (3) is defined in a very similar way, with only $\|x(t)\|^{2}$ being replaced by $\|x(t)\|$.

Definition 2 The trivial solution of system (3) is said to be asymptotically mean square stable on the interval $[0, \infty)$ if it is stable and, moreover,

$$
\lim _{t \rightarrow \infty} E^{(1)}\left\{\|x(t)\|^{2}\right\}=0
$$

is satisfied for each solution of (3).

Remark 1 It is easy to see that (4) is satisfied if and only if the matrix $E^{(2)}\{x(t)\}$ converges to zero matrix $\Theta$,

$$
\lim _{t \rightarrow \infty} E^{(2)}\{x(t)\}=\lim _{t \rightarrow \infty} E^{(1)}\left\{x(t) x^{T}(t)\right\}=\Theta .
$$

Denote a neighborhood of the point $o \in \mathbb{R}^{m}$ as $\mathcal{O}(o)$.

Definition 3 The function $g(t, x(t))$ is said to be positive definite on $\mathcal{O}(o)$ if $g(t, x)$ is continuous with respect to $t, t \geq 0,\|x(t)\|<\infty$ on $\mathcal{O}(o), g(t, o)=0$, and there exists a positive definite quadratic form $V(x)$ such that

$$
g(t, x) \geq V(x) \quad \text { whenever } t \geq 0, \quad x \in \mathcal{O}(o) \text { and }\|x(t)\|<\infty .
$$

Recall, if a function $g(t, x(t))$ is positive definite on $\mathcal{O}(o)$, then the function $-g(t, x(t))$ is negative definite on this neighborhood. 
Remark 2 The previous definition is equivalent to the following one:

The function $g(t, x(t))$ is said to be positive definite on $\mathcal{O}(o)$ if $g(t, x)$ is continuous with respect to $t, t \geq 0,\|x(t)\|<\infty$ on $\mathcal{O}(o), g(t, o)=0$, and there exists a constant $k>0$ such that for any positive definite quadratic form $V(x)$,

$$
g(t, x) \geq k V(x) \quad \text { whenever } t \geq 0, \quad x \in \mathcal{O}(o) \text { and }\|x(t)\|<\infty
$$

\section{Main results}

We define a Lyapunov function $v$ in the form

$$
v(x(t))=x^{T}(t) C x(t),
$$

where $C$ is an $m \times m$ positive definite symmetric matrix. We also use the diagonal matrix $C_{d}$ which has the same elements on the diagonal as $C$.

Theorem 1 Let there exist a neighborhood $\mathcal{O}(o)$, in which the function

$$
\delta(x)=2 x^{T} C f(x)+h^{T}(x) C_{d} h(x)
$$

is negative definite with respect to system (3).

Then the trivial solution of (3) is asymptotically mean square stable on the interval $[0, \infty)$

Proof First we calculate the differential of Lyapunov function (5) with respect to trajectories of system (3). We have

$$
\begin{aligned}
d v(x)= & v(x+d x)-v(x)=\left(x^{T}+d x^{T}\right) C(x+d x)-x^{T} C x \\
= & \left(x^{T}+f^{T}(x) d t+(h(x) d w(t))^{T}\right) C(x+f(x) d t+(h(x) d w(t)))-x^{T} C x \\
= & x^{T} C x-x^{T} C x+x^{T} C f(x) d t+x^{T} C(h(x) d w(t))+f^{T}(x) d t C x \\
& +f^{T}(x) d t C f(x) d t+f^{T}(x) d t C(h(x) d w(t))+(h(x) d w(t))^{T} C x \\
& +(h(x) d w(t))^{T} C f(x) d t+(h(x) d w(t))^{T} C(h(x) d w(t)) .
\end{aligned}
$$

After modifying the obtained equation, applying operation mathematical expectation $E^{(1)}\{d v(t)\}$, in regard to assumptions (2), and leaving aside the member with $d t^{2}$, we get the equation

$$
E^{(1)}\{d v(x)\}=x^{T} C f(x) d t+f^{T}(x) C x d t+h^{T}(x) C_{d} h(x) d t,
$$

or, in view of (6), it can be written in the more simple form

$$
E^{(1)}\{d v(x)\}=\delta(x) d t
$$

Because $\delta(x)$ is a negative definite deterministic function, the equalities

$$
\begin{aligned}
& E^{(1)}\{\delta(x)\}=\delta(x), \\
& \delta(x) \leq-k v(x), \quad k=\mathrm{const},
\end{aligned}
$$


are true (see Remark 2). By using them and equation (8), we get

$$
\frac{d}{d t} E^{(1)}\{v(x)\} \leq-k E^{(1)}\{v(x)\}
$$

so

$$
E^{(1)}\{v(x)\} \leq e^{-k t} .
$$

Therefore,

$$
\lim _{t \rightarrow \infty} E^{(2)}\{x(t)\}=\lim _{t \rightarrow \infty} E^{(1)}\left\{x(t) x^{T}(t)\right\}=\Theta,
$$

which implies asymptotic mean square stability of a trivial solution of the considered system.

Remark 3 Analogous result about instability can be derived in the same way as the result of Theorem 1 .

Namely, if there exists a neighborhood $\mathcal{O}(o)$, in which the function

$$
\delta(x)=2 x^{T} C f(x)+h^{T}(x) C_{d} h(x)
$$

is positive definite with respect to system (3), then the trivial solution of (3) is unstable on the interval $[0, \infty)$.

Further we will discuss the stability of a system in the more general form

$$
d x(t)=f(t, x) d t+\sum_{k=1}^{n} h_{k}(t, x) d w_{k}(t), \quad t \geq 0,
$$

where $f, h_{k}, k=1, \ldots, n$, are $m$-dimensional column vector-functions continuously differentiable in both variables, such that $f(t, o)=o, h_{k}(t, o)=o$ hold. Expressions $h_{k}(t, x) d w_{k}(t)$, $k=1, \ldots, n$, are again column vectors with components $h_{i k}(t, x) d w_{i k}(t), i=1,2, \ldots, m$, $k=1,2, \ldots, n$. Functions $w_{k}=\left(w_{1 k}, \ldots, w_{m k}\right)^{T}, k=1,2, \ldots, n$, are also $m$-dimensional column vector-functions indicating standard Wiener processes, such that each of them satisfies the following relationships:

$$
\begin{aligned}
& w_{k}(0)=o, \\
& E^{(1)}\left\{d w_{k}(t)\right\}=o, \\
& E^{(1)}\left\{d w_{k}(t) d w_{k}^{T}(t)\right\}=I d t, \quad k=1,2, \ldots, n
\end{aligned}
$$

for $t \geq 0$.

The following theorem can be proved in the same way as Theorem 1 .

Theorem 2 Let there exist a neighborhood $\mathcal{O}(o)$, in which the function

$$
\delta(t, x)=2 x^{T} C f(t, x)+\sum_{k=1}^{n} h_{k}^{T}(t, x) C_{d} h_{k}(t, x)
$$

is negative definite with respect to system (9). 
Then the trivial solution of (9) is asymptotically mean square stable on the interval $[0, \infty)$.

Remark 4 A similar remark as Remark 3 can be formulated in this case. Namely, if there exists a neighborhood $\mathcal{O}(o)$, in which the function

$$
\delta(t, x)=2 x^{T} C f(t, x)+\sum_{k=1}^{n} h_{k}^{T}(t, x) C_{d} h_{k}(t, x)
$$

is positive definite with respect to system (9), then the trivial solution of (9) is unstable on the interval $[0, \infty)$.

In the last part of this section, let us consider one special case of a stochastic system of differential equations, namely the linear system

$$
d x(t)=A x(t) d t+H x(t) d w(t), \quad t \geq 0,
$$

where $A, H$ are $m \times m$ constant matrices and $w$ is a standard Wiener process that satisfies (2).

Corollary 1 Let there exist a neighborhood $\mathcal{O}(o)$, in which the function

$$
\delta(x)=x^{T} A^{T} C x+x^{T} C A x+x^{T} H^{T} C_{d} H x
$$

is negative definite with respect to system (12).

Then the trivial solution of (12) is asymptotically mean square stable on the interval $[0, \infty)$

Proof The proof of this theorem follows immediately from Theorem 1 if $f(x)=A x, h(x)=$ $H x$.

Remark 5 If there exists a neighborhood $\mathcal{O}(o)$, in which the function

$$
\delta(x)=x^{T} A^{T} C x+x^{T} C A x+x^{T} H^{T} C_{d} H x
$$

is positive definite with respect to system (12), then the trivial solution of (12) is unstable on the interval $[0, \infty)$.

Remark 6 Stability of the trivial solution of a stochastic system of linear differential equations in the form (12) was studied in the works by Korenevskii in [7]. The following stability criterion was obtained there by different methods: The trivial solution of system (12) is asymptotically mean square stable on the interval $[0, \infty)$ if and only if there exists a positive definite matrix $C$ satisfying the matrix equation

$$
A^{T} C+C A+H^{T} C H=-G,
$$

where matrix $G$ is positive definite. 


\section{Examples}

In the following examples, we illustrate application of Theorem 1 and Remark 3. In these examples, we also construct the corresponding Lyapunov function; however, it is not necessary for verifying the stability or instability condition.

Example 1 We consider stochastic functional differential equation in the form

$$
d x(t)=-x(t) d t-x^{3}(t) d w(t)
$$

where $f, h$ are scalar functions, $f=-x(t), h=-x^{3}(t), x$ is a scalar state function and $w$ is a standard Wiener process. Stability of the zero solution of this equation can be determined on the basis of Theorem 1 .

We define Lyapunov function in the form (5), with $C=1$,

$$
v(x)=x^{2}
$$

Then equation (7) is in the form

$$
E^{(1)}\{d v(t)\}=\left(-x^{T} x-x^{T} x+\left(x^{3}\right)^{T} x^{3}\right) d t=\left(-2 x^{2}+x^{6}\right) d t .
$$

To apply Theorem 1 , we have to prove that there exists a neighborhood of the zero point 0 , where the function $\delta=-2 x^{2}+x^{6}$ is negative definite. This holds if and only if the following inequality is satisfied:

$$
x^{6}-2 x^{2}<0 \quad \Leftrightarrow \quad 0<x^{4}<2
$$

or

$$
-\sqrt[4]{2}<x<\sqrt[4]{2}, \quad x \neq 0
$$

That is, $\delta$ is the function of negative values at each point of $(-\sqrt[4]{2}, \sqrt[4]{2})$ except zero, and $\delta(0)=0$. Therefore, we have the conclusion that there exists a neighborhood $\mathcal{O}(0)=$ $(-\sqrt[4]{2}, \sqrt[4]{2})$ in which the function $\delta=-2 x^{2}+x^{6}$ is negative definite. So, the trivial solution $x=0$ of considered equation is asymptotically mean square stable on the interval $[0, \infty)$.

Example 2 We consider stochastic differential equation in the form

$$
d x(t)=a x(t) d t+b x(t) d w(t)
$$

where $a, b \in \mathbb{R}, a \neq 0$ and $x$ is a scalar state function.

We again define Lyapunov function in the form (5) with $C=1$. The function $\delta$, in view of Theorem 1, has the form

$$
\delta(x)=\left(2 a+b^{2}\right) x^{2} .
$$

Hence, if $2 a+b^{2}<0$, the function $\delta$ is negative definite, in the opposite case it is positive definite. Therefore, in accordance with Theorem 1 and Remark 3, if $2 a+b^{2}<0$, then the 
trivial solution of considered equation is asymptotically mean square stable on the interval $[0, \infty)$. In the opposite case, that is, if $2 a+b^{2}>0$, then the trivial solution of considered equation is unstable.

Example 3 We consider stochastic functional differential equation in the form

$$
d x(t)=-x(t) d t+a \sin x(t) d w(t)
$$

where $f, g$ are scalar functions, $f=-x(t), g=a \sin x(t), a \in \mathbb{R}, a \neq 0$ and $x$ is a scalar state function.

We again define Lyapunov function in the form (5) with $C=1$. Therefore, a function $\delta$ from (6) has the form

$$
\begin{aligned}
\delta(t, x) & =-2 x^{T} x+(a \sin x(t))^{T} a \sin x(t) \\
& =-2 x^{2}+a^{2} \sin ^{2} x .
\end{aligned}
$$

Because

$$
-2 x^{2}+a^{2} \sin ^{2} x>0 \Leftrightarrow \frac{x^{2}}{\sin ^{2} x}<\frac{a^{2}}{2}
$$

in the case $a>\sqrt{2}$, there exists a neighborhood of the zero point 0 , where the function $\delta$ is positive definite. In the case $a \leq \sqrt{2}$, the function $\delta$ is negative definite. So, the zero solution of considered equation is unstable for any $a>\sqrt{2}$ and it is asymptotically mean square stable for any $a \leq \sqrt{2}$.

Example 4 Now we consider two-dimensional stochastic functional differential equation in the form

$$
\begin{aligned}
& d x_{1}(t)=x_{2}(t) d t+h_{1}\left(x_{1}, x_{2}\right) d w(t), \\
& d x_{2}(t)=-x_{1}(t) d t+h_{2}\left(x_{1}, x_{2}\right) d w(t),
\end{aligned}
$$

where $x=\left(x_{1}, x_{2}\right)^{T}$ is a two-dimensional vector state function, $f, h$ are two-dimensional vector functions, $f=\left(x_{2}(t),-x_{1}(t)\right)^{T}, h=\left(h_{1}\left(x_{1}, x_{2}\right), h_{2}\left(x_{1}, x_{2}\right)\right)^{T}, h \neq(0,0)$ on a neighborhood $\mathcal{O}(0,0)$ with the exception of the zero point $(0,0)$, and $w$ is a standard Wiener process.

We again define Lyapunov function in the form (5) with $C=I$,

$$
v(x)=x_{1}^{2}+x_{2}^{2}
$$

Then the function $\delta=h^{T} h$ and it is positive definite on the neighborhood $\mathcal{O}(0,0)$. Thus, by Theorem 1 , the trivial zero solution of this equation is unstable on the interval $[0, \infty)$.

Remark 7 If we take $h_{1}=0$ in system (17), then it represents the stochastic second order differential equation

$$
d^{2} x(t)=-x(t) d t+h_{2}\left(x^{\prime}, x\right) d w(t)
$$


and the previous example implies that its solution is unstable for any nontrivial $h_{2}$. But a function can be added to the right-hand side of this equation such that the trivial solution of the new equation will be asymptotically mean square stable. The following result holds:

If

$$
\lim _{\left(x_{1}, x_{2}\right) \rightarrow(0,0)} \frac{g\left(x_{1}, x_{2}\right) \cdot x_{2}}{h_{2}^{2}\left(x_{1}, x_{2}\right)}=L>\frac{1}{2}
$$

for a continuously differentiable function $g\left(x_{1}, x_{2}\right)$, then the trivial solution of the system

$$
\begin{aligned}
& d x_{1}(t)=x_{2}(t) d t \\
& d x_{2}(t)=-x_{1}(t) d t-g\left(x_{1}, x_{2}\right) d t+h_{2}\left(x_{1}, x_{2}\right) d w(t)
\end{aligned}
$$

is asymptotically mean square stable.

Condition (18) holds, for example, for $g\left(x_{1}, x_{2}\right)=h_{2}\left(x_{1}, x_{2}\right)=x_{2}$. Then system (19) is the following:

$$
\begin{aligned}
& d x_{1}(t)=x_{2}(t) d t, \\
& d x_{2}(t)=-x_{1}(t) d t-x_{2}(t) d t+x_{2}(t) d w(t) .
\end{aligned}
$$

Behavior of solutions $x_{1}(t)$ of this system compared to that of solutions of the unstable system

$$
\begin{aligned}
& d x_{1}(t)=x_{2}(t) d t \\
& d x_{2}(t)=-x_{1}(t) d t+x_{2}(t) d w(t)
\end{aligned}
$$

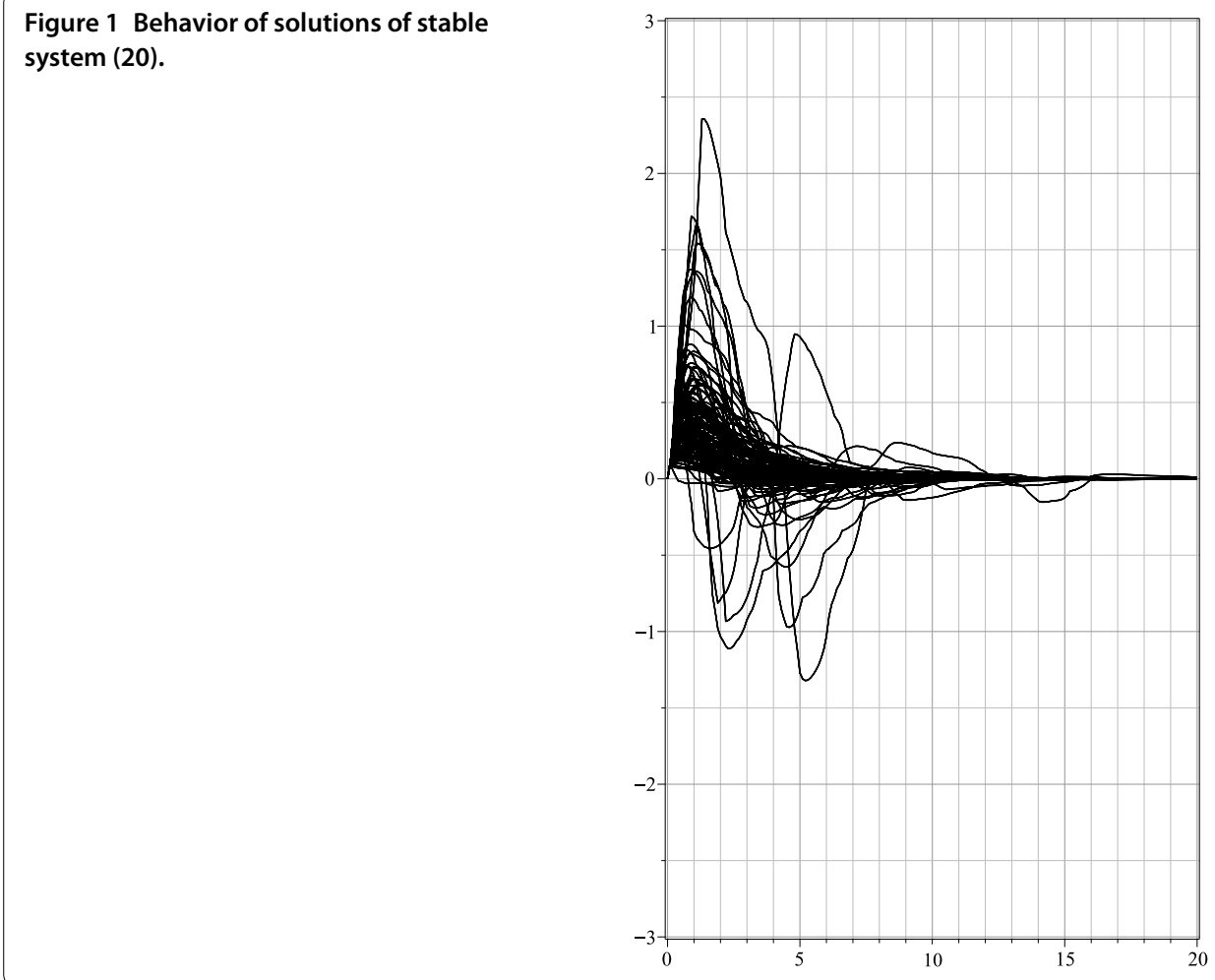




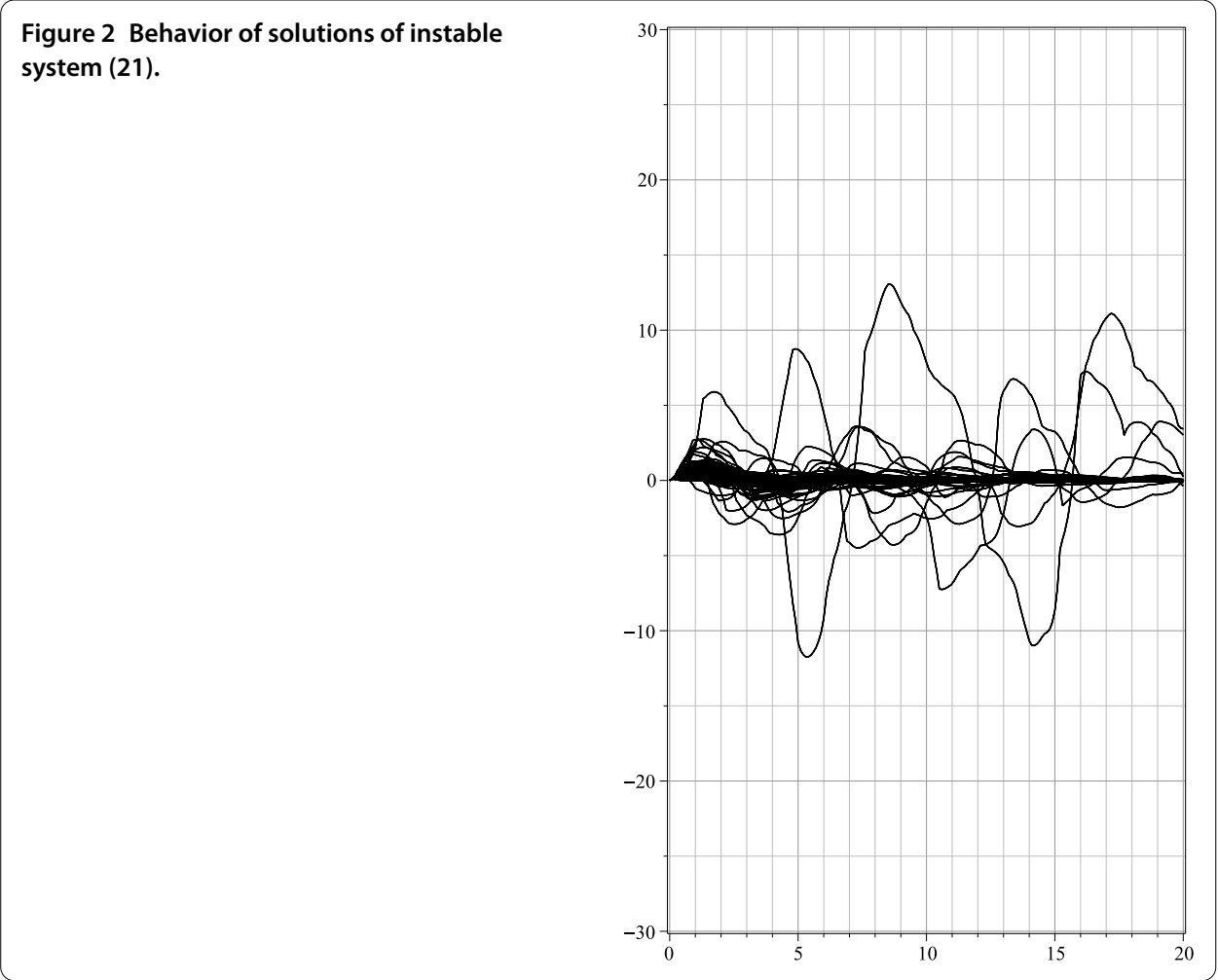

is illustrated in Figures 1 and 2. In both of these figures, there are displayed 120 solutions of the systems (20), (21) with initial conditions $x_{1}(0)=0, x_{2}(0)=1$, respectively. These solutions were computed numerically with the Milstein method on the interval $[0,20]$.

\section{Conclusion}

Our concept of studying asymptotic mean square stability of the zero solution of stochastic differential equations is more effective in comparison with previously known methods, and it can also be used in various application problems. For example, by stochastic equation of the type

$$
d x(t)=\left[a_{1} x(t)-a_{2} x(t) \ln x(t)\right] d t+\sigma x(t) d w(t)
$$

we can describe a tumor growth, where the expected size of the tumor is contaminated with white noise [8]. Such type of equations can be used also in biomedical research [9, 10], epidemic modeling [11], in describing animal motion [12], receiving signals [10, 13] and many others. However, the approach to the study of these models in the cited works is different compared to our method. They use numerical or statistical methods and estimation methods in there. 


\section{Author details}

${ }^{1}$ Kiev National Economics Vadym Hetman University, Kyiv, Ukraine. ${ }^{2}$ University of Žilina, Žilina, Slovakia. ${ }^{3}$ Brno University of Technology, Brno, Czech Republic.

\section{Acknowledgements}

The second author was supported by Grant KEGA 004ŽU-4/2014 of Slovak Grant Agency. The third author was supported by the project FSI-S-14-2290 of Brno University of Technology.

Received: 11 February 2015 Accepted: 22 April 2015 Published online: 07 May 2015

\section{References}

1. Hasselmann, K: Stochastic climate models. Part I: Theory. Tellus 28, 473-485 (1976)

2. Arnold, L: Hasselmann's program visited: the analysis of stochasticity in deterministic climate models. In: von Storch, J-S, Imkeller, P (eds.) Stochastic Climate Models, pp. 141-158. Birkhäuser, Boston (2001)

3. Dymnikov, VP, Lykosov, NV, Volodin, EM: Problems of modeling climate and climate change. IzV., Atmos. Ocean. Phys. 42(5), 568-585 (2006)

4. Benzi, R, Sutera, A, Vulpiani, A: The mechanism of stochastic resonance. J. Phys. A, Math. Gen. 14(11), L453-L457 (1981)

5. Benzi, R, Parisi, G, Sutera, A, Vulpiani, A: A theory of stochastic resonance in climatic change. SIAM J. Appl. Math. 43(3), 565-578 (1983)

6. Lipster, R, Shiryaev, A: Statistics of Random Processes I: General Theory, 2nd edn. Springer, Berlin (2001)

7. Korenevskii, DH: The Stability of Solutions to the System of Differential Equations with Coefficients Perturbed by White Noise and Coloured Noises. IM of NASU, Kiev (2012) (in Russian)

8. Lo, CF: Stochastic nonlinear Gompertz model of tumour growth. In: Proceedings of the World Congress on Engineering, London, UK, 1-3 July (2009)

9. Donnet, S, Samson, A: A review on estimation of stochastic differential equations for pharmacokinetic/pharmacodynamic models. Adv. Drug Deliv. Rev. 65(7), 929-939 (2013)

10. Picchini, U, Ditlevsen, S, De Gaetano, A, Lansky, P: Parameters of the diffusion leaky integrate-and-fire neuronal model for a slowly fluctuating signal. Neural Comput. 20(11), 2696-2714 (2008)

11. Rao, F: Dynamics analysis of a stochastic SIR epidemic model. Abstr. Appl. Anal. 2014, Article ID 356013 (2014)

12. Brillinger, DR, Preisler, HK, Ager, AA, Kie, JG, Stewart, BS: Employing stochastic differential equations to model wildlife motion. Bull. Braz. Math. Soc. 33(3), 385-408 (2002)

13. Gardiner, CW: Handbook of Stochastic Methods for Physics, Chemistry and the Natural Sciences, 2nd edn. Springer, Berlin (1985)

\section{Submit your manuscript to a SpringerOpen ${ }^{\ominus}$ journal and benefit from:}

- Convenient online submission

- Rigorous peer review

- Immediate publication on acceptance

Open access: articles freely available online

- High visibility within the field

- Retaining the copyright to your article 